\title{
Common fixed point of a power graphic contraction pair in partial metric spaces endowed with a graph
}

\author{
Mujahid Abbas ${ }^{1}$ and Talat Nazir ${ }^{*}$
}

*Correspondence: talat@ciit.net.pk 2Department of Mathematics, COMSATS Institute of Information Technology, Abbottabad, 22060, Pakistan

Full list of author information is available at the end of the article

\begin{abstract}
In this paper, we initiate a study of fixed point results in the setup of partial metric spaces endowed with a graph. The concept of a power graphic contraction pair of two mappings is introduced. Common fixed point results for such maps without appealing to any form of commutativity conditions defined on a partial metric space endowed with a directed graph are obtained. These results unify, generalize and complement various known comparable results from the current literature.

MSC: $47 \mathrm{H} 10 ; 54 \mathrm{H} 25 ; 54 \mathrm{E} 50$
\end{abstract}

Keywords: partial metric space; common fixed point; directed graph; power graphic contraction pair

\section{Introduction and preliminaries}

Consistent with Jachymski [1], let $X$ be a nonempty set and $d$ be a metric on $X$. A set $\{(x, x): x \in X\}$ is called a diagonal of $X \times X$ and is denoted by $\Delta$. Let $G$ be a directed graph such that the set $V(G)$ of its vertices coincides with $X$ and $E(G)$ is the set of the edges of the graph with $\Delta \subseteq E(G)$. Also assume that the graph $G$ has no parallel edges. One can identify a graph $G$ with the pair $(V(G), E(G))$. Throughout this paper, the letters $\mathbb{R}, \mathbb{R}^{+}, \omega$ and $\mathbb{N}$ will denote the set of real numbers, the set of nonnegative real numbers, the set of nonnegative integers and the set of positive integers, respectively.

Definition 1.1 [1] A mapping $f: X \rightarrow X$ is called a Banach G-contraction or simply Gcontraction if

$\left(\mathrm{a}_{1}\right)$ for each $x, y \in X$ with $(x, y) \in E(G)$, we have $(f(x), f(y)) \in E(G)$,

$\left(\mathrm{a}_{2}\right)$ there exists $\alpha \in(0,1)$ such that for all $x, y \in X$ with $(x, y) \in E(G)$ implies that $d(f(x), f(y)) \leq \alpha d(x, y)$.

Let $X^{f}:=\{x \in X:(x, f(x)) \in E(G)$ or $(f(x), x) \in E(G)\}$.

Recall that if $f: X \rightarrow X$, then a set $\{x \in X: x=f(x)\}$ of all fixed points of $f$ is denoted by $F(f)$. A self-mapping $f$ on $X$ is said to be

(1) a Picard operator if $F(f)=\left\{x^{*}\right\}$ and $f^{n}(x) \rightarrow x^{*}$ as $n \rightarrow \infty$ for all $x \in X$;

(2) a weakly Picard operator if $F(f) \neq \emptyset$ and for each $x \in X$, we have $f^{n}(x) \rightarrow x^{*} \in F(f)$ as $n \rightarrow \infty$;

(c) 2013 Abbas and Nazir; licensee Springer. This is an Open Access article distributed under the terms of the Creative Commons Attribution License (http://creativecommons.org/licenses/by/2.0), which permits unrestricted use, distribution, and reproduction in any medium, provided the original work is properly cited. 
(3) orbitally continuous if for all $x, a \in X$, we have

$$
\lim _{k \rightarrow \infty} f^{n_{k}}(x)=a \quad \text { implies } \quad \lim _{i \rightarrow \infty} f\left(f^{n_{k}}(x)\right)=f(a)
$$

The following definition is due to Chifu and Petrusel [2].

Definition 1.2 An operator $f: X \rightarrow X$ is called a Banach $G$-graphic contraction if

$\left(\mathrm{b}_{1}\right)$ for each $x, y \in X$ with $(x, y) \in E(G)$, we have $(f(x), f(y)) \in E(G)$,

$\left(b_{2}\right)$ there exists $\alpha \in[0,1)$ such that

$$
d\left(f(x), f^{2}(x)\right) \leq \alpha d(x, f(x)) \text { for all } x \in X^{f}
$$

If $x$ and $y$ are vertices of $G$, then a path in $G$ from $x$ to $y$ of length $k \in \mathbb{N}$ is a finite sequence $\left\{x_{n}\right\}, n \in\{0,1,2, \ldots, k\}$ of vertices such that $x_{0}=x, x_{k}=y$ and $\left(x_{i-1}, x_{i}\right) \in E(G)$ for $i \in\{1,2, \ldots, k\}$.

Notice that a graph $G$ is connected if there is a path between any two vertices and it is weakly connected if $\widetilde{G}$ is connected, where $\widetilde{G}$ denotes the undirected graph obtained from $G$ by ignoring the direction of edges. Denote by $G^{-1}$ the graph obtained from $G$ by reversing the direction of edges. Thus,

$$
E\left(G^{-1}\right)=\{(x, y) \in X \times X:(y, x) \in E(G)\} .
$$

Since it is more convenient to treat $\widetilde{G}$ as a directed graph for which the set of its edges is symmetric, under this convention, we have that

$$
E(\widetilde{G})=E(G) \cup E\left(G^{-1}\right)
$$

If $G$ is such that $E(G)$ is symmetric, then for $x \in V(G)$, the symbol $[x]_{G}$ denotes the equivalence class of the relation $R$ defined on $V(G)$ by the rule:

$y R z$ if there is a path in $G$ from $y$ to $z$.

A graph $G$ is said to satisfy the property (A) (see also [2]) if for any sequence $\left\{x_{n}\right\}$ in $V(G)$ with $x_{n} \rightarrow x$ as $n \rightarrow \infty$ and $\left(x_{n}, x_{n+1}\right) \in E(G)$ for $n \in \mathbb{N}$ implies that $\left(x_{n}, x\right) \in E(G)$.

Jachymski [1] obtained the following fixed point result for a mapping satisfying the Banach $G$-contraction condition in metric spaces endowed with a graph.

Theorem 1.3 [1] Let $(X, d)$ be a complete metric space and $G$ be a directed graph and let the triple $(X, d, G)$ have a property (A). Let $f: X \rightarrow X$ be a G-contraction. Then the following statements hold:

1. $F_{f} \neq \emptyset$ if and only if $X_{f} \neq \emptyset$;

2. if $X_{f} \neq \emptyset$ and $G$ is weakly connected, then $f$ is a Picard operator;

3. for any $x \in X_{f}$ we have that $\left.f\right|_{[x]_{\widetilde{G}}}$ is a Picard operator;

4. iff $\subseteq E(G)$, then $f$ is a weakly Picard operator. 
Gwodzdz-Lukawska and Jachymski [3] developed the Hutchinson-Barnsley theory for finite families of mappings on a metric space endowed with a directed graph. Bojor [4] obtained a fixed point of a $\varphi$-contraction in metric spaces endowed with a graph (see also [5]). For more results in this direction, we refer to [2, 6, 7].

On the other hand, Mathews [8] introduced the concept of a partial metric to obtain appropriate mathematical models in the theory of computation and, in particular, to give a modified version of the Banach contraction principle more suitable in this context. For examples, related definitions and work carried out in this direction, we refer to [9-19] and the references mentioned therein. Abbas et al. [20] proved some common fixed points in partially ordered metric spaces (see also [21]). Gu and He [22] proved some common fixed point results for self-maps with twice power type $\Phi$-contractive condition. Recently, Gu and Zhang [23] obtained some common fixed point theorems for six self-mappings with twice power type contraction condition.

Throughout this paper, we assume that a nonempty set $X=V(G)$ is equipped with a partial metric $p$, a directed graph $G$ has no parallel edge and $G$ is a weighted graph in the sense that each vertex $x$ is assigned the weight $p(x, x)$ and each edge $(x, y)$ is assigned the weight $p(x, y)$. As $p$ is a partial metric on $X$, the weight assigned to each vertex $x$ need not be zero and whenever a zero weight is assigned to some edge $(x, y)$, it reduces to a loop $(x, x)$.

Also, the subset $W(G)$ of $V(G)$ is said to be complete if for every $x, y \in W(G)$, we have $(x, y) \in E(G)$.

Definition 1.4 Self-mappings $f$ and $g$ on $X$ are said to form a power graphic contraction pair if

(a) for every vertex $v$ in $G,(v, f v)$ and $(v, g v) \in E(G)$,

(b) there exists $\phi: \mathbb{R}^{+} \rightarrow \mathbb{R}^{+}$an upper semi-continuous and nondecreasing function with $\phi(t)<t$ for each $t>0$ such that

$$
p^{\delta}(f x, g y) \leq \phi\left(p^{\alpha}(x, y) p^{\beta}(x, f x) p^{\gamma}(y, g y)\right)
$$

for all $(x, y) \in E(G)$ holds, where $\alpha, \beta, \gamma \geq 0$ with $\delta=\alpha+\beta+\gamma \in(0, \infty)$.

If we take $f=g$, then the mapping $f$ is called a power graphic contraction.

The aim of this paper is to investigate the existence of common fixed points of a power graphic contraction pair in the framework of complete partial metric spaces endowed with a graph. Our results extend and strengthen various known results $[8,12,13,24]$.

\section{Common fixed point results}

We start with the following result.

Theorem 2.1 Let $(X, p)$ be a complete partial metric space endowed with a directed graph G. If $, g: X \rightarrow X$ form a power graphic contraction pair, then the following hold:

(i) $F(f) \neq \emptyset$ or $F(g) \neq \emptyset$ if and only if $F(f) \cap F(g) \neq \emptyset$.

(ii) If $u \in F(f) \cap F(g)$, then the weight assigned to the vertex $u$ is 0 .

(iii) $F(f) \cap F(g) \neq \emptyset$ provided that $G$ satisfies the property (A).

(iv) $F(f) \cap F(g)$ is complete if and only if $F(f) \cap F(g)$ is a singleton. 
Proof To prove (i), let $u \in F(f)$. By the given assumption, $(u, g u) \in E(G)$. Assume that we assign a non-zero weight to the edge $(u, g u)$. As $(u, u) \in E(G)$ and $f$ and $g$ form a power graphic contraction, we have

$$
\begin{aligned}
p^{\delta}(u, g u) & =p^{\delta}(f u, g u) \\
& \leq \phi\left(p^{\alpha}(u, u) p^{\beta}(u, f u) p^{\gamma}(u, g u)\right) \\
& =\phi\left(p^{\alpha+\beta}(u, u) p^{\gamma}(u, g u)\right) \\
& \leq \phi\left(p^{\alpha+\beta}(u, g u) p^{\gamma}(u, g u)\right) \\
& =\phi\left(p^{\delta}(u, g u)\right) \\
& <p^{\delta}(u, g u),
\end{aligned}
$$

a contradiction. Hence, the weight assigned to the edge $(u, g u)$ is zero and so $u=g u$. Therefore, $u \in F(f) \cap F(g) \neq \emptyset$. Similarly, if $u \in F(g)$, then we have $u \in F(f)$. The converse is straightforward.

Now, let $u \in F(f) \cap F(g)$. Assume that the weight assigned to the vertex $u$ is not zero, then from (1.1), we have

$$
\begin{aligned}
p^{\delta}(u, u) & =p^{\delta}(f u, g u) \\
& \leq \phi\left(p^{\alpha}(u, u) p^{\beta}(u, f u) p^{\gamma}(u, g u)\right) \\
& =\phi\left(p^{\alpha+\beta+\gamma}(u, u)\right) \\
& =\phi\left(p^{\delta}(u, u)\right) \\
& <p^{\delta}(u, u),
\end{aligned}
$$

a contradiction. Hence, (ii) is proved.

To prove (iii), we will first show that there exists a sequence $\left\{x_{n}\right\}$ in $X$ with $f x_{2 n}=x_{2 n+1}$ and $g x_{2 n+1}=x_{2 n+2}$ for all $n \in \mathbb{N}$ with $\left(x_{n}, x_{n+1}\right) \in E(G)$, and $\lim _{n \rightarrow \infty} p\left(x_{n}, x_{n+1}\right)=0$.

Let $x_{0}$ be an arbitrary point of $X$. If $f x_{0}=x_{0}$, then the proof is finished, so we assume that $f x_{0} \neq x_{0}$. As $\left(x_{0}, f x_{0}\right) \in E(G)$, so $\left(x_{0}, x_{1}\right) \in E(G)$. Also, $\left(x_{1}, g x_{1}\right) \in E(G)$ gives $\left(x_{1}, x_{2}\right) \in E(G)$. Continuing this way, we define a sequence $\left\{x_{n}\right\}$ in $X$ such that $\left(x_{n}, x_{n+1}\right) \in E(G)$ with $f x_{2 n}=$ $x_{2 n+1}$ and $g x_{2 n+1}=x_{2 n+2}$ for $n \in \mathbb{N}$.

We may assume that the weight assigned to each edge $\left(x_{2 n}, x_{2 n+1}\right)$ is non-zero for all $n \in \mathbb{N}$. If not, then $x_{2 k}=x_{2 k+1}$ for some $k$, so $f x_{2 k}=x_{2 k+1}=x_{2 k}$, and thus $x_{2 k} \in F(f)$. Hence, $x_{2 k} \in F(f) \cap F(g)$ by (i). Now, since $\left(x_{2 n}, x_{2 n+1}\right) \in E(G)$, so from (1.1), we have

$$
\begin{aligned}
p^{\delta}\left(x_{2 n+1}, x_{2 n+2}\right) & =p^{\delta}\left(f x_{2 n}, g x_{2 n+1}\right) \\
& \leq \phi\left(p^{\alpha}\left(x_{2 n}, x_{2 n+1}\right) p^{\beta}\left(x_{2 n}, f x_{2 n}\right) p^{\gamma}\left(x_{2 n+1}, g x_{2 n+1}\right)\right) \\
& =\phi\left(p^{\alpha}\left(x_{2 n}, x_{2 n+1}\right) p^{\beta}\left(x_{2 n}, x_{2 n+1}\right) p^{\gamma}\left(x_{2 n+1}, x_{2 n+2}\right)\right) \\
& =\phi\left(p^{\alpha+\beta}\left(x_{2 n}, x_{2 n+1}\right) p^{\gamma}\left(x_{2 n+1}, x_{2 n+2}\right)\right) \\
& <p^{\alpha+\beta}\left(x_{2 n}, x_{2 n+1}\right) p^{\gamma}\left(x_{2 n+1}, x_{2 n+2}\right),
\end{aligned}
$$


which implies that

$$
p^{\alpha+\beta}\left(x_{2 n+1}, x_{2 n+2}\right)<p^{\alpha+\beta}\left(x_{2 n}, x_{2 n+1}\right) \text {, }
$$

a contradiction if $\alpha+\beta=0$. So, take $\alpha+\beta>0$, and we have

$$
p\left(x_{2 n+1}, x_{2 n+2}\right)<p\left(x_{2 n}, x_{2 n+1}\right)
$$

for all $n \in \mathbb{N}$. Again from (1.1), we have

$$
\begin{aligned}
p^{\delta}\left(x_{2 n+2}, x_{2 n+3}\right) & =p^{\delta}\left(g x_{2 n+1}, f x_{2 n+2}\right) \\
& =p^{\delta}\left(f x_{2 n+2}, g x_{2 n+1}\right) \\
& \leq \phi\left(p^{\alpha}\left(x_{2 n+2}, x_{2 n+1}\right) p^{\beta}\left(x_{2 n+2}, f x_{2 n+2}\right) p^{\gamma}\left(x_{2 n+1}, g x_{2 n+1}\right)\right) \\
& =\phi\left(p^{\alpha}\left(x_{2 n+1}, x_{2 n+2}\right) p^{\beta}\left(x_{2 n+2}, x_{2 n+3}\right) p^{\gamma}\left(x_{2 n+1}, x_{2 n+2}\right)\right) \\
& =\phi\left(p^{\alpha+\gamma}\left(x_{2 n+1}, x_{2 n+2}\right) p^{\beta}\left(x_{2 n+2}, x_{2 n+3}\right)\right) \\
& <p^{\alpha+\gamma}\left(x_{2 n+1}, x_{2 n+2}\right) p^{\beta}\left(x_{2 n+2}, x_{2 n+3}\right)
\end{aligned}
$$

which implies that

$$
p^{\alpha+\gamma}\left(x_{2 n+2}, x_{2 n+3}\right)<p^{\alpha+\gamma}\left(x_{2 n+1}, x_{2 n+2}\right) .
$$

We arrive at a contradiction in case $\alpha+\gamma=0$. Therefore, we must take $\alpha+\gamma>0$; consequently, we have

$$
p\left(x_{2 n+2}, x_{2 n+3}\right)<p\left(x_{2 n+1}, x_{2 n+2}\right)
$$

for all $n \in \mathbb{N}$. Hence,

$$
p^{\delta}\left(x_{n}, x_{n+1}\right) \leq \phi\left(p^{\delta}\left(x_{n-1}, x_{n}\right)\right)<p^{\delta}\left(x_{n-1}, x_{n}\right)
$$

for all $n \in \mathbb{N}$. Therefore, the decreasing sequence of positive real numbers $\left\{p^{\delta}\left(x_{n}, x_{n+1}\right)\right\}$ converges to some $c \geq 0$. If we assume that $c>0$, then from (2.1) we deduce that

$$
0<c \leq \limsup _{n \rightarrow \infty} \phi\left(p^{\delta}\left(x_{n-1}, x_{n}\right)\right) \leq \phi(c)<c,
$$

a contradiction. So, $c=0$, that is, $\lim _{n \rightarrow \infty} p^{\delta}\left(x_{n}, x_{n+1}\right)=0$ and so we have $\lim _{n \rightarrow \infty} p\left(x_{n}\right.$, $\left.x_{n+1}\right)=0$. Also,

$$
p^{\delta}\left(x_{n}, x_{n+1}\right) \leq \phi\left(p^{\delta}\left(x_{n-1}, x_{n}\right)\right) \leq \cdots \leq \phi^{n}\left(p^{\delta}\left(x_{0}, x_{1}\right)\right) .
$$

Now, for $m, n \in \mathbb{N}$ with $m>n$,

$$
\begin{aligned}
p^{\delta}\left(x_{n}, x_{m}\right) \leq & p^{\delta}\left(x_{n}, x_{n+1}\right)+p^{\delta}\left(x_{n+1}, x_{n+2}\right)+\cdots+p^{\delta}\left(x_{m-1}, x_{m}\right) \\
& -p^{\delta}\left(x_{n+1}, x_{n+1}\right)-p^{\delta}\left(x_{n+2}, x_{n+2}\right)-\cdots-p^{\delta}\left(x_{m-1}, x_{m-1}\right) \\
\leq & \phi^{n}\left(p^{\delta}\left(x_{0}, x_{1}\right)\right)+\phi^{n+1}\left(p^{\delta}\left(x_{0}, x_{1}\right)\right)+\cdots+\phi^{m-1}\left(p^{\delta}\left(x_{0}, x_{1}\right)\right)
\end{aligned}
$$


implies that $p^{\delta}\left(x_{n}, x_{m}\right)$ converges to 0 as $n, m \rightarrow \infty$. That is, $\lim _{n, m \rightarrow \infty} p\left(x_{n}, x_{m}\right)=0$. Since $(X, p)$ is complete, following similar arguments to those given in Theorem 2.1 of [9], there exists a $u \in X$ such that $\lim _{n, m \rightarrow \infty} p\left(x_{n}, x_{m}\right)=\lim _{n \rightarrow \infty} p\left(x_{n}, u\right)=p(u, u)=0$. By the given hypothesis, $\left(x_{2 n}, u\right) \in E(G)$ for all $n \in \mathbb{N}$. We claim that the weight assigned to the edge $(u, g u)$ is zero. If not, then as $f$ and $g$ form a power graphic contraction, so we have

$$
\begin{aligned}
p^{\delta}\left(x_{2 n+1}, u\right) & =p^{\delta}\left(f x_{2 n}, g u\right) \\
& \leq \phi\left(p^{\alpha}\left(x_{2 n}, u\right) p^{\beta}\left(x_{2 n}, f x_{2 n}\right) p^{\gamma}(u, g u)\right) \\
& =\phi\left(p^{\alpha}\left(x_{2 n}, u\right) p^{\beta}\left(x_{2 n}, x_{2 n+1}\right) p^{\gamma}(u, g u)\right) .
\end{aligned}
$$

We deduce, by taking upper limit as $n \rightarrow \infty$ in (2.3), that

$$
\begin{aligned}
p^{\delta}(u, g u) & \leq \limsup _{n \rightarrow \infty} \phi\left(p^{\alpha}\left(x_{2 n}, u\right) p^{\beta}\left(x_{2 n}, x_{2 n+1}\right) p^{\gamma}(u, g u)\right) \\
& \leq \phi\left(p^{\alpha}(u, u) p^{\beta}(u, u) p^{\gamma}(u, g u)\right) \\
& \leq \phi\left(p^{\alpha+\beta+\gamma}(u, g u)\right) \\
& <p^{\delta}(u, g u)
\end{aligned}
$$

a contradiction. Hence, $u=g u$ and $u \in F(f) \cap F(g)$ by (i).

Finally, to prove (iv), suppose the set $F(f) \cap F(g)$ is complete. We are to show that $F(f) \cap$ $F(g)$ is a singleton. Assume on the contrary that there exist $u$ and $v$ such that $u, v \in F(f) \cap$ $F(g)$ but $u \neq v$. As $(u, v) \in E(G)$ and $f$ and $g$ form a power graphic contraction, so

$$
\begin{aligned}
0 & <p^{\delta}(u, v)=p^{\delta}(f u, f v) \\
& \leq \phi\left(p^{\alpha}(u, v) p^{\beta}(u, f u) p^{\gamma}(v, g v)\right) \\
& =\phi\left(p^{\alpha}(u, v) p^{\beta}(u, u) p^{\gamma}(v, v)\right) \\
& \leq \phi\left(p^{\delta}(u, v)\right),
\end{aligned}
$$

a contradiction. Hence, $u=v$. Conversely, if $F(f) \cap F(g)$ is a singleton, then it follows that $F(f) \cap F(g)$ is complete.

Corollary 2.2 Let $(X, p)$ be a complete partial metric space endowed with a directed graph $G$. If we replace (1.1) by

$$
p^{\delta}\left(f^{s} x, g^{t} y\right) \leq \phi\left(p^{\alpha}(x, y) p^{\beta}\left(x, f^{s} x\right) p^{\gamma}\left(y, g^{t} y\right)\right)
$$

where $\alpha, \beta, \gamma \geq 0$ with $\delta=\alpha+\beta+\gamma \in(0, \infty)$ and $s, t \in \mathbb{N}$, then the conclusions obtained in Theorem 2.1 remain true.

Proof It follows from Theorem 2.1, that $F\left(f^{s}\right) \cap F\left(g^{t}\right)$ is a singleton provided that $F\left(f^{s}\right) \cap$ $F\left(g^{t}\right)$ is complete. Let $F\left(f^{s}\right) \cap F\left(g^{t}\right)=\{w\}$, then we have $f(w)=f\left(f^{s}(w)\right)=f^{s+1}(w)=f^{s}(f(w))$, and $g(w)=g\left(g^{t}(w)\right)=g^{t+1}(w)=g^{t}(g(w))$ implies that $f w$ and $g w$ are also in $F\left(f^{s}\right) \cap F\left(g^{t}\right)$. Since $F\left(f^{s}\right) \cap F\left(g^{t}\right)$ is a singleton, we deduce that $w=f w=g w$. Hence, $F(f) \cap F(g)$ is a singleton. 
The following remark shows that different choices of $\alpha, \beta$ and $\gamma$ give a variety of power graphic contraction pairs of two mappings.

Remarks 2.3 Let $(X, p)$ be a complete partial metric space endowed with a directed graph $G$.

(R1) We may replace (1.1) with the following:

$$
p^{3}(f x, g y) \leq \phi(p(x, y) p(x, f x) p(y, g y))
$$

to obtain conclusions of Theorem 2.1. Indeed, taking $\alpha=\beta=\gamma=1$ in Theorem 2.1, one obtains (2.5).

(R2) If we replace (1.1) by one of the following condition:

$$
\begin{aligned}
& p^{2}(f x, g y) \leq \phi(p(x, y) p(x, f x)), \\
& p^{2}(f x, g y) \leq \phi(p(x, y) p(y, g y)), \\
& p^{2}(f x, g y) \leq \phi(p(x, f x) p(y, g y)),
\end{aligned}
$$

then the conclusions obtained in Theorem 2.1 remain true. Note that

(i) if we take $\alpha=\beta=1$ and $\gamma=0$ in (1.1), then we obtain (2.6),

(ii) take $\alpha=\gamma=1, \beta=0$ in (1.1) to obtain (2.7),

(iii) use $\beta=\gamma=1, \alpha=0$ in (1.1) and obtain (2.8).

(R3) Also, if we replace (1.1) by one of the following conditions:

$$
\begin{aligned}
& p(f x, g y) \leq \phi(p(x, y)), \\
& p(f x, g y) \leq \phi(p(x, f x)), \\
& p(f x, g y) \leq \phi(p(y, g y)),
\end{aligned}
$$

then the conclusions obtained in Theorem 2.1 remain true. Note that

(iv) take $\alpha=1$ and $\beta=\gamma=0$ in (1.1) to obtain (2.9),

(v) to obtain (2.10), take $\beta=1, \alpha=\gamma=0$ in (1.1),

(vi) if one takes $\gamma=1, \alpha=\beta=0$ in (1.1), then we obtain (2.11).

Remark 2.4 If we take $f=g$ in a power graphic contraction pair, then we obtain fixed point results for a power graphic contraction.

Competing interests

The authors declare that they have no competing interests.

Authors' contributions

All authors read and approved the final manuscript.

\section{Author details}

${ }^{1}$ Department of Mathematics, Lahore University of Management Sciences, Lahore, 54792, Pakistan. ${ }^{2}$ Department of Mathematics, COMSATS Institute of Information Technology, Abbottabad, 22060, Pakistan. 


\section{References}

1. Jachymski, J: The contraction principle for mappings on a metric space with a graph. Proc. Am. Math. Soc. 136 1359-1373 (2008)

2. Chifu, Cl, Petrusel, GR: Generalized contractions in metric spaces endowed with a graph. Fixed Point Theory Appl. 2012, 161 (2012). doi:10.1186/1687-1812-2012-161

3. Gwozdz-Lukawska, G, Jachymski, J: IFS on a metric space with a graph structure and extensions of the Kelisky-Rivlin theorem. J. Math. Anal. Appl. 356, 453-463 (2009)

4. Bojor, F: Fixed point of $\varphi$-contraction in metric spaces endowed with a graph. An. Univ. Craiova, Ser. Math. Comput. Sci. 37(4), 85-92 (2010)

5. Bojor, F: Fixed point theorems for Reich type contractions on metric spaces with a graph. Nonlinear Anal. 75, 3895-3901 (2012)

6. Aleomraninejad, SMA, Rezapoura, S, Shahzad, N: Some fixed point results on a metric space with a graph. Topol. Appl. $159,659-663(2012)$

7. Nicolae, A, O'Regan, D, Petrusel, A: Fixed point theorems for singlevalued and multivalued generalized contractions in metric spaces endowed with a graph. Georgian Math. J. 18, 307-327 (2011)

8. Matthews, SG: Partial metric topology. In: Proc. 8th Summer Conference on General Topology and Applications. Ann. New York Acad. Sci., vol. 728, pp. 183-197 (1994)

9. Abbas, M, Nazir, T: Fixed point of generalized weakly contractive mappings in ordered partial metric spaces. Fixed Point Theory Appl. 2012, 1 (2012)

10. Abbas, M, Nazir, T, Romaguera, S: Fixed point results for generalized cyclic contraction mappings in partial metric spaces. Rev. R. Acad. Cienc. Exactas Fís. Nat., Ser. A Mat. (2011). doi:10.1007/s13398-011-0051-5

11. Altun, I, Simsek, H: Some fixed point theorems on dualistic partial metric spaces. J. Adv. Math. Stud. 1, 1-8 (2008)

12. Altun, I, Sola, F, Simsek, H: Generalized contractions on partial metric spaces. Topol. Appl. 157, 2778-2785 (2010)

13. Altun, I, Erduran, A: Fixed point theorems for monotone mappings on partial metric spaces. Fixed Point Theory Appl. 2011, Article ID 508730 (2011)

14. Bukatin, MA, Shorina, SY: Partial metrics and co-continuous valuations. In: Nivat, M (ed.) Foundations of Software Science and Computation Structure. Lecture Notes in Computer Science, vol. 1378, pp. 125-139. Springer, Berlin (1998)

15. Bukatin, M, Kopperman, R, Matthews, S, Pajoohesh, H: Partial metric spaces. Am. Math. Mon. 116, 708-718 (2009)

16. Oltra, S, Valero, O: Banach's fixed theorem for partial metric spaces. Rend. Ist. Mat. Univ. Trieste 36, 17-26 (2004)

17. Romaguera, S: A Kirk type characterization of completeness for partial metric spaces. Fixed Point Theory Appl. 2010, Article ID 493298 (2010)

18. Schellekens, MP: The correspondence between partial metrics and semivaluations. Theor. Comput. Sci. 315, 135-149 (2004)

19. Valero, O: On Banach fixed point theorems for partial metric spaces. Appl. Gen. Topol. 6, 229-240 (2005)

20. Abbas, M, Nazir, T, Radenović, S: Common fixed points of four maps in partially ordered metric spaces. Appl. Math. Lett. 24, 1520-1526 (2011)

21. Altun, I, Simsek, H: Some fixed point theorems on ordered metric spaces and application. Fixed Point Theory Appl. 2010, Article ID 621492 (2010)

22. Gu, F, He, Z: The common fixed point theorems for a class of twice power type $\Phi$-contraction mapping. J. Shangqiu Teach. Coll. 22(5), 27-33 (2006)

23. Gu, F, Zhang, D: The common fixed point theorems for six self mappings with twice power type contraction condition. Thai J. Math. 10(3), 587-603 (2012)

24. Aydi, H: Some fixed point results in ordered partial metric spaces (2011). arxiv:1103.3680v1 [math.GN]

doi:10.1186/1687-1812-2013-20

Cite this article as: Abbas and Nazir: Common fixed point of a power graphic contraction pair in partial metric spaces endowed with a graph. Fixed Point Theory and Applications 2013 2013:20.

\section{Submit your manuscript to a SpringerOpen ${ }^{\circ}$ journal and benefit from:}

- Convenient online submission

- Rigorous peer review

- Immediate publication on acceptance

Open access: articles freely available online

- High visibility within the field

- Retaining the copyright to your article 